\title{
ANL/XFD/CP-92700
}

\section{Thermal contact resistance across a copper-silicon interface}

\author{
A.M. Khounsary, D. Chojnowski, and L. Assoufid \\ Advanced Photon Source \\ Experimental Facilities Division \\ Argonne National Laboratory, Argonne, IL 60439-4843 \\ W. M. Worek \\ University of Illinois at Chicago \\ Department of Mechanical Engineering \\ Chicago, Illinois 60607
}

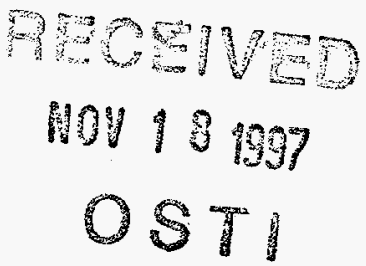

\begin{abstract}
An experimental setup to measure the thermal contact conductance across a silicon-copper ( $\mathrm{Si}-\mathrm{Cu}$ ) interface is described, and the results obtained are presented. The resulting thermal contact resistance data are used in estimating the thermo-mechanical and optical performance of optical substrates cooled by interfaced copper cooling blocks.

Several factors influence the heat transfer across solid interfaces. These include the material properties, interface pressure, flatness and roughness of the contacting surfaces, temperature, and interstitial material, if any.

Results presented show the variation of thermal contact conductance as a function of applied interface pressure for a $\mathrm{Cu}$-Si interface. Various interstitial materials investigated include indium foil, silver foil and a liquid eutectic (Ga-In-Sn). As expected, thermal contact resistance decreases as interface pressure increases, except in the case of the eutectic, in which it was nearly constant. The softer the interstitial material, the lower the thermal contact resistance. Liquid metal provides the lowest thermal contact resistance across the $\mathrm{Cu}$-Si interface, followed by the indium foil, and then the silver foil.
\end{abstract}

keywords: contact resistance, thermal interface, heat transfer.

\section{INTRODUCTION}

The issue of thermal contact resistance across metallic interfaces has been investigated for many situations over the past several decades (Yovanovich and Antonetti, 1988; Lambert and Fletcher, 1997). In most cases the investigation has focused on obtaining data useful to researchers for a particular application. Often in published reports, certain important experimental parameters have been left out, creating uncertainty and making it difficult to use or apply the data to other situations. The study reported here was done due to the lack of published data needed for our application.

The application in the present case is contact cooling of high heat load optical substrates. High heat load $\mathrm{x}$-ray mirrors and other optical components used at the Advanced Photon Source (APS) are either internally cooled or contact cooled. In the internally cooled mirrors, a coolant flows through passages configured in the optical substrate. In the contactcooled case, cooling is provided by placing cooling plates in contact with the mirror to extract the heat.

One of the issues in the contact-cooled mirror design is the thermal resistance across the cooling plate-optical substrate interface. At the APS, these materials are often copper and silicon, respectively. Other hard substrate materials, such as silicon carbide or diamond, behave similarly to $\mathrm{Si}$ when in contact with copper (or softer materials) because the softer material often determines the interface behavior. Thermal resistance in practical terms (or its inverse, the conductance) depends on (1) material hardness, (2) roughness and flatness of the surfaces, (3) interstitial material, if any, (4) applied interface force, (5) thermal conditions, and (6) other less significant factors.

Many optical substrates at the APS operate in high vacuum (HV) or ultrahigh vacuum (UHV) environments. Thus, an attempt is made to estimate the copper-silicon thermal contact conductance $h_{c}$ in vacuum with silver foil, indium, and GaIn-Sn eutectic. 


\section{DISCLAIMER}

This report was prepared as an account of work sponsored by an agency of the United States Government. Neither the United States Government nor any agency thereof, nor any of their employees, makes any warranty, express or implied, or assumes any legal liability or responsibility for the accuracy, completeness, or usefulness of any information, apparatus, product, or process disclosed, or represents that its use would not infringe privately owned rights. Reference herein to any specific commercial product, process, or service by trade name, trademark, manufacturer, or otherwise does not necessarily constitute or imply its endorsement, recommendation, or favoring by the United States Government or any agency thereof. The views and opinions of authors expressed herein do not necessarily state or reflect those of the United States Government or any agency thereof. 


\section{DESClaAmERR}

Portions of this docoment may be illegible in electronic image products. Irrages are produced from the best available original doerment. 
The relevance of conductance data to the present application is better understood if one notes that higher thermal contact conductance will (a) reduce the overall substrate temperature, (b) reduce the mirror time constant (i.e., faster approach to thermal equilibrium), and (c) reduce the thermal distortion in the substrate (distortion in silicon increases by an additional $1 \%$ for every $1^{\circ} \mathrm{C}$ rise in the temperature due to nonlinearity in $\mathrm{k} / \alpha$, where $\mathrm{k}$ and $\alpha$ are the thermal conductivity and expansion coefficient, respectively).

Thermal contact conductance at the $\mathrm{Si}-\mathrm{Cu}$ interface can be improved by increasing the actual contact area. This can be done by one or more of the following: (1) making copper and silicon contact surfaces very smooth and flat, (2) applying a high pressure at the interface, and (3) using a soft interstitial material (soft metals such as indium, liquid metals, or a variety of conductive greases or liquids).

There are limitations to each one of these options, operational as well as practical. In the first case, for example, highly smooth and flat surfaces can be costly to produce and tend to bond together when placed in contact. Applying a very high interface pressure can actually distort the optical surface. The third solution appears to be the simplest and most straightforward. The use of a soft interstitial material to fill some of the surface voids and irregularities, in conjunction with a light to moderate interface pressure, can increase the heat conductance across the substrate-cooling block boundary by as much as an order of magnitude or more.

Here an experimental setup was devised and used to obtain thermal contact resistance for Si-Cu interfaces in a vacuum environment. Thermal conductance at the interface of a copper and a silicon cylinder was obtained for the mentioned interstitial materials, at several interface pressures.

\section{EXPERIMENTAL SETUP}

The experimental setup is illustrated by Figure 1 and Figure 2. Figure 1 shows the overall vacuum test chamber, while Figure 2 illustrates the test cylinder column setup.

The interface force is applied to the test column by a bellows attached to the top plate (see Figure 3 ). The inside of the bellows is tapped into the vacuum pump line and is also ported to the atmosphere with ball valves for pressure control. By opening or closing the appropriate valve, interface pressure can be gradually increased to a maximum pressure by creating maximum pressure differential between the bellows chamber and the vacuum chamber.

A Pressure plate lowering shaft

B Aluminum top plate, $35 \mathrm{~cm} \times 35 \mathrm{~cm} \times 2 \mathrm{~cm}$

C 3 viton gaskets between the plates, the collar and the glass cylinder

D Glass cylinder, 12" diameter, protected by a steelmesh cage

E Feedthrough collar

F 2 water feedthroughs

G 3 feedthroughs for a sum of 18 thermocouples

$\mathrm{H}$ Aluminum base plate

I Bellows

J Thermal insulator

$\mathrm{K}$ Thermocouple wires

L Cold Plate

$\mathrm{M}$ Load cell

$\mathrm{N}$ Bellows chamber

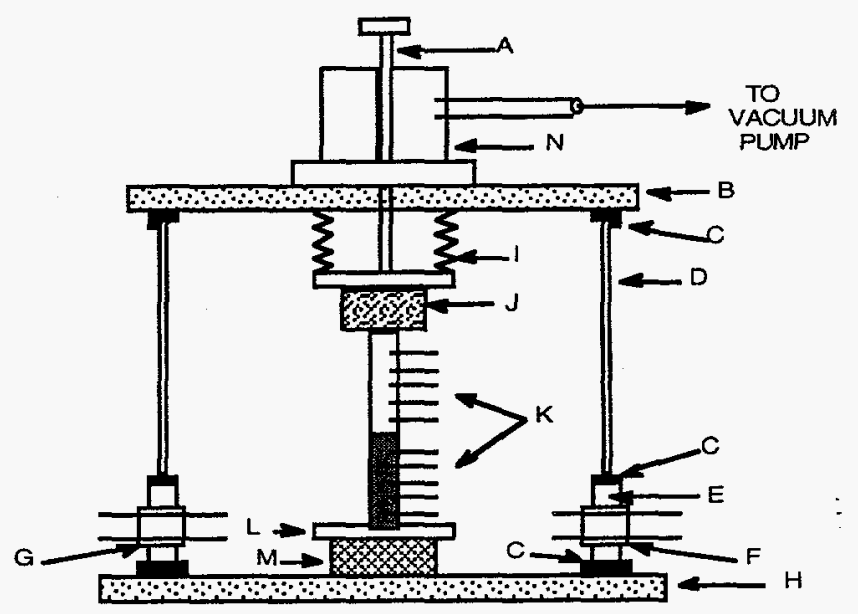

Figure 1: Vacuum test chamber used and its components.

The chamber is evacuated to a pressure of $13 \mathrm{~Pa}(0.1$ Torr) to significantly reduce heat transfer by convection in the system (Kennard, 1938).

The chamber basically consists of a $30 \mathrm{~cm}$ (12") diameter glass cylinder and a stainless steel feedthrough port ring sandwiched between 2-cm-thick aluminum plates. The feedthrough ring is a $13 \mathrm{~cm}\left(5^{\prime \prime}\right)$ tall and 30 (12") diameter with 12 ports distributed evenly around the perimeter. The top and bottom surfaces have gasket seating surfaces to seal against the glass cylinder and the bottom plate. Four threaded rods align and compress the assembly, creating the vacuum chamber. 


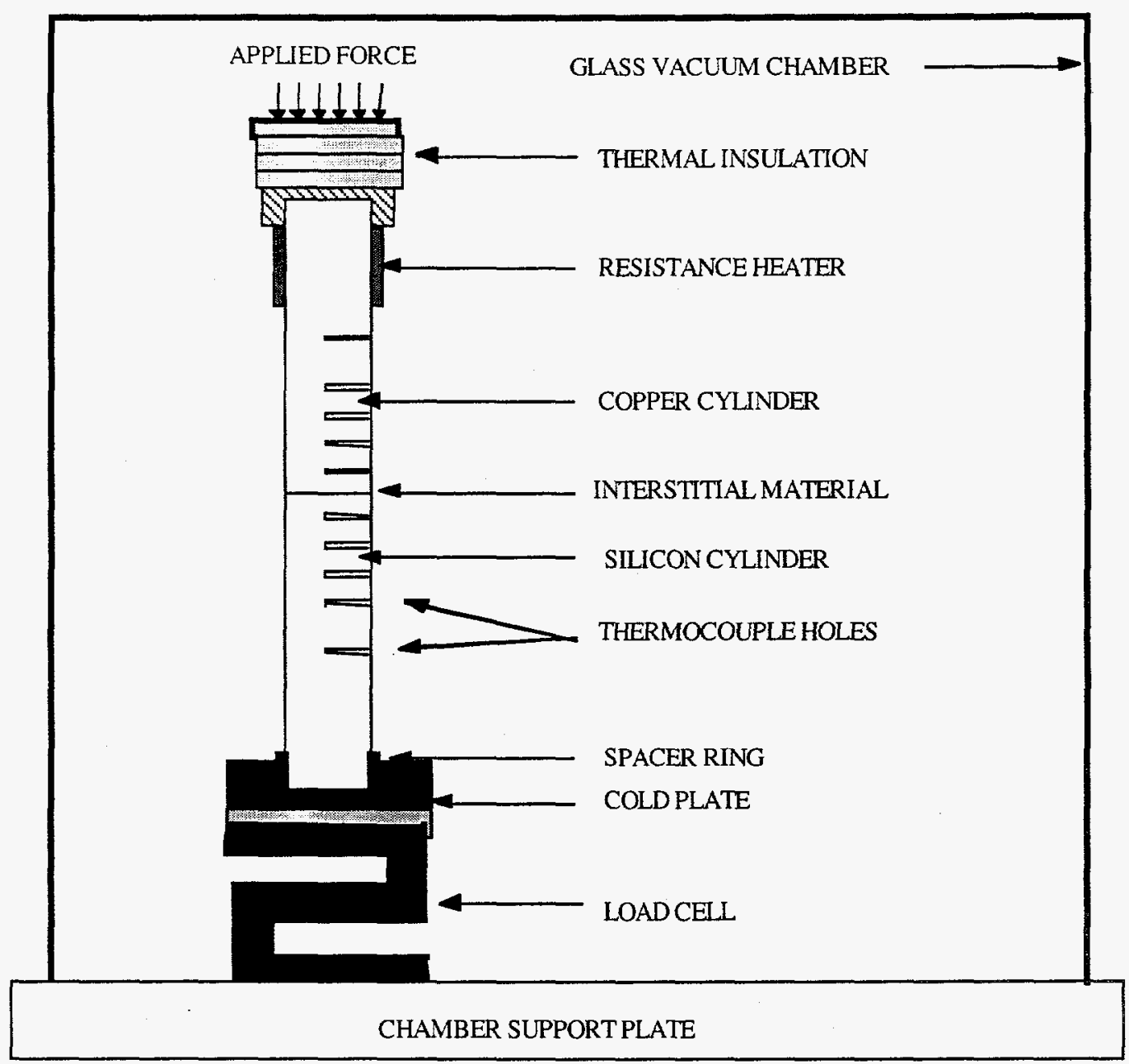

Figure 2: Test column used in the thermal contact conductance experiment.

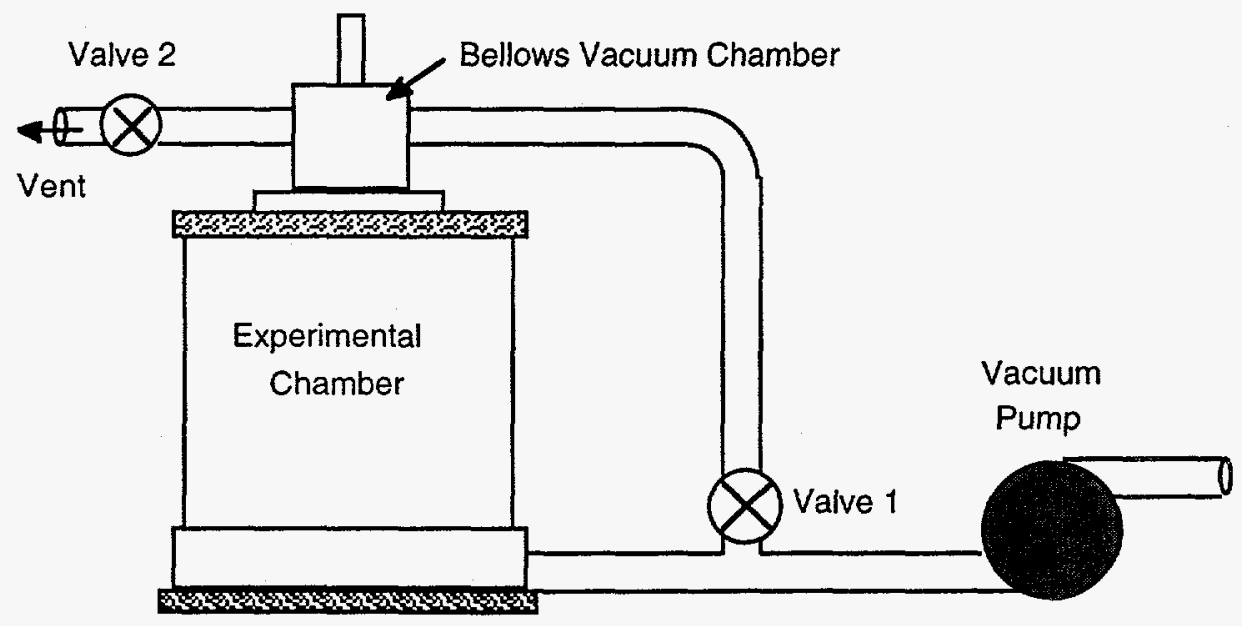

Figure 3: Vacuum system components and piping. 
The 12 ports are used for thermocouples, electrical power wires for the heater, load cell signal wires, vacuum pump connection, water cooling lines for cold plate, and vacuum gauge connections.

The thermal insulation indicated in Figure 2 is G-10 fiberglass laminate pieces. This combination of thick and thin $4.6 \mathrm{~cm}\left(1.8^{\prime \prime}\right)$ square laminates creates a reasonable thermal resistance to reduce the heat flux flowing upward from the heater. A thermocouple is embedded in the thickest laminate piece to monitor the temperature rise during experimental runs.

The thermocouples used in this experiment are type K, stainless-steel sheathed, ungrounded thermocouples. The three vacuum port feedthroughs provide 18 thermocouples, 15 of which were used. Of those, 12 are used to measure the temperatures of the test cylinders, one is used to monitor the temperature of the cooled base in which the silicon cylinder is mounted, and the other two measure temperatures, respectively, of the G-10 and stainless steel pieces above the heated copper cylinder. The other three thermocouples inside the chamber are spares.

Before the thermocouples were installed, they were tested and calibrated for temperatures in the $25-140^{\circ} \mathrm{C}$ range at 5 or $10^{\circ} \mathrm{C}$ intervals. The procedure used a constant temperature bath (with microprocessor controller) that had a temperature stability of $\pm 0.01{ }^{\circ} \mathrm{C}$. For calibration temperatures under $90^{\circ} \mathrm{C}$, water was used, and for those above $90^{\circ} \mathrm{C}$, peanut oil was used. Precision, NIST-certified, partial immersion thermometers were also placed in the bath for a reference of the actual temperature. This procedure resulted in an thermocouple temperature accuracy of $+/-0.1^{\circ} \mathrm{C}$.

The data acquisition system consisted of a PC-based thermocouple system. A visual graphing and acquisition program was developed to record and also plot thermocouple temperatures in real time. Typically, it took a few hours for the system to reach steady state condition.

\section{THEORY}

When heat flows through two bodies placed in contact, there is a macroscopic temperature discontinuity at the interface. This temperature discontinuity results from thermal contact resistance. For two surfaces in contact, even those that are visually very smooth and flat, the actual contact area is typically less than $10 \%$ of the total area. It is at these areas that the heat flows from one body to the other. Because of heat transfer paths, the heat flux is therefore not uniform in the bodies near the interface. At some distance further away from the interface, the heat flux distributes throughout the materials and can be considered as one-dimensional heat flow. Because of the nonhomogeneous temperature distribution in a cross-sectional area, cylinder temperature measurements should not be taken close to the interface. It is due to this behavior that the thermocouples in the experiment are not positioned closer than $5 \mathrm{~mm}$ from the interface. Figure 4 illustrates the placement of the thermocouples along the test cylinders, along with the accompanying plot of temperatures used to determine the contact resistance. As the illustration shows, a straight line is fit to the temperatures for each cylinder. The $x=0$ location refers to the location of the interface. The temperature vs. location plots are extrapolated to the interface, giving a temperature drop $(\Delta \mathrm{T})$ due to the contact resistance.

The two temperature vs. location lines plotted result in values for $\mathrm{dT} / \mathrm{dx}$ for each cylinder. Using this slope in Fourier's law and the materials thermal conductivity $(\mathrm{k})$, a value for the heat flux flowing through the cylinders is obtained:

$$
q=-k \frac{d T}{d x}
$$

Because the thermal conductivity of a material is temperature dependent, the value of $\mathrm{k}$ corresponding to each cylinder's average measured temperature is used.

If no losses occur as heat travels down the cylinders and across the interface, then the calculated $q$ for both cylinders should be equal. However, losses do occur, and the $\mathrm{q}$ for the silicon cylinder is slightly less than the $\mathrm{q}$ for the copper cylinder in all cases. The sources of these losses include radiation from the cylinders, free molecular conduction to the gases remaining in the chamber, and conduction through the thermocouple wires.

As will be shown from the resulting data, the difference in the calculated heat flux $\left(q_{c u}-q_{s i}\right)$ measured in each cylinder averages about $5 \%$. Because the sum of these losses is small, further investigation of these individual losses is not necessary.

The thermal contact conductance is determined from the measurements of flux through the cylinders and the predicted (extrapolated) temperature drop across the interface. The relation used is:

$$
\text { Conductance }=h=\frac{q}{\Delta T}
$$



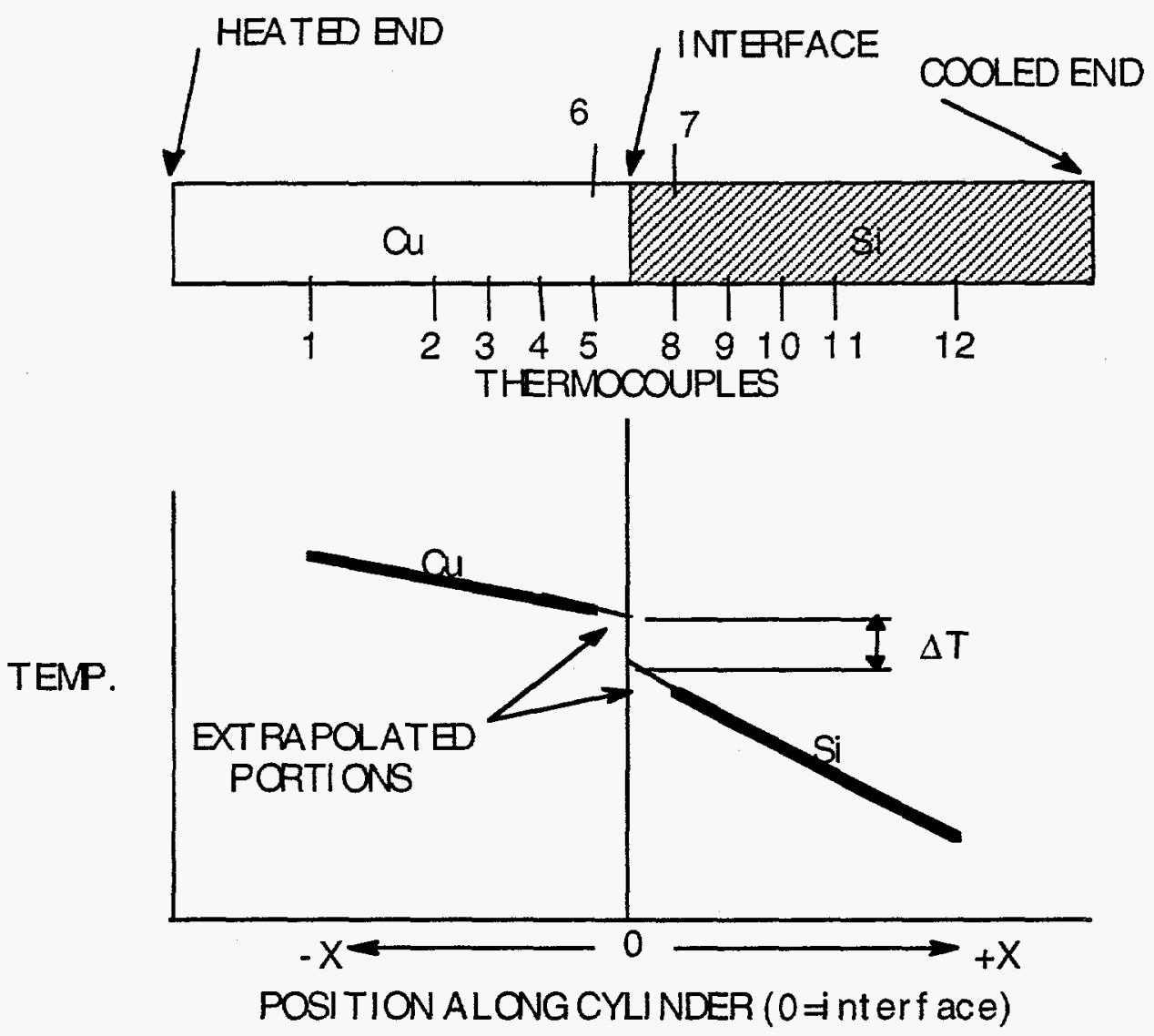

Figure 4: Illustration of the test cylinders with 12 thermocouples (top) and temperature drop across the interface (bottom).

Because the measured $\mathrm{q}$ for the top cylinder and the measured $\mathrm{q}$ for the bottom cylinder are slightly different $(\approx 5 \%)$, the arithmetic average of the two is used for the conductance calculation.

One OFHC (oxygen-free high conductivity) copper cylinder and one single-crystal-type silicon cylinder, each $25 \mathrm{~mm}$ ( 1 inch) in diameter, were used for all of the conductance tests. Table 1 lists select contact surface parameters measured for each cylinder with a Mitutoyo Surftest 301 surface profiler. Surface parameter readings were repeated at many different orientations and averaged to arrive at the results below.

Table 1 - Surface Characteristics of Test Cylinders

\begin{tabular}{|c|l|c|c|}
\hline PARAMETER & \multicolumn{1}{|c|}{ DEFINITION } & Cu CYLINDER & Si CYLINDER \\
\hline $\mathrm{R}_{\mathrm{a}}$ & Roughness average & $0.07 \mu \mathrm{m}$ & $0.09 \mu \mathrm{m}$ \\
\hline $\mathrm{R}_{\mathrm{t}}$ & Maximum peak-to-valley height & $0.7 \mu \mathrm{m}$ & $1.7 \mu \mathrm{m}$ \\
\hline $\mathrm{L}(\lambda)$ & Evaluation lengths & $0.25,0.8,2.5 \mathrm{~mm}$ & $0.25,0.8,2.5 \mathrm{~mm}$ \\
\hline
\end{tabular}

Three different types of interstitial material were investigated. These consisted of indium foil of thickness $0.1 \mathrm{~mm}$, 0.28 -mm-thick silver foil, and a gallium-indium-tin eutectic. The eutectic was mixed in the following proportions: $65 \% \mathrm{Ga}$, $25 \%$ In, $10 \% \mathrm{Sn}$. The liquid metal was applied by rubbing the eutectic into both cylinder surfaces until they were 'wet' by the fluid.

After each incremented run from zero to maximum interface force, the cylinders were separated, and the foil was replaced after thorough cleaning of both cylinder surfaces. Care was taken to make sure cylinders were realigned to the same exact orientations as before. 
Table 2 summarizes the number of experimental runs for each of the interstitial materials used and the experimental conditions tested.

Table 2 - Experimental Run Parameters Tested

\begin{tabular}{|c|c|c|c|}
\hline $\begin{array}{c}\text { INTERSTITIAL } \\
\text { MATERIAL }\end{array}$ & $\begin{array}{c}\text { POWER INPUT TEST } \\
\text { POINTS (Watts) }\end{array}$ & $\begin{array}{c}\text { INTERFACE FORCES } \\
\text { TESTED }(\mathrm{kg})\end{array}$ & $\begin{array}{c}\text { NUMBER OF TESTS } \\
\text { RUN }\end{array}$ \\
\hline Indium foil & $5,9,10,20,40$ & $8,12,20,40,60$ & 46 \\
\hline Silver foil & $10,20,30,40$ & $8,12,20,40,60$ & 32 \\
\hline Eutectic & $10,20,40$ & $8,20,60$ & 8 \\
\hline
\end{tabular}

Fewer eutectic experimental runs were completed due to the fact that varying interface force has little effect on resulting conductance, as liquid fills much of the surface voids without the addition of interface force.

\section{RESULTS AND DISCUSSION}

All experimental runs were started at minimum interface pressure and incremented to maximum interface pressure. Figure 5 summarizes the results, showing the variations of thermal contact conductance with pressure for the three interstitial materials. As the figure indicates, thermal contact conductance increases as interface pressure is increased because of increased deformation of interstitial material leading to larger contact area. Figure 5 also shows that the contact conductance with the eutectic generally is not a function of interface pressure. This is due to the liquid form of the eutectic. When interface force is increased, little increase in contact area is made, and no significant change in interstitial conductance occurs.

From Figure 5, one sees that the slopes vary for the three interface materials (note the logarithmic y-axis). The conductance slope is greatest for indium and less for silver. The curve for the eutectic interstitial material, as expected, is flat, indicating little change in conductance with in interface pressure.

A simple error analysis can be performed to estimate the error in the results. It is noted that in the calculation of the thermal contact conductance, two basic relations are used. First is Fourier's law:

$$
q=-k \frac{d T}{d x}
$$

where $\mathrm{q}, \mathrm{k}, \mathrm{T}$, and $\mathrm{x}$ are the heat flux, thermal conductivity, temperature, and distance, respectively.

The second equation used to compute the thermal contact conductance is simply the heat flux divided by the temperature drop across the interface, $\Delta \mathrm{T}$ :

$$
h=\frac{q}{\Delta T} .
$$

An error analysis consisting of examining the sources of errors in each of these quantities has been carried out. Errors arise from inaccuracies in temperature measurements, thermocouple location, thermal conductivity data used, heat flux measurements, and estimation of the temperature drop across the interface using a linear temperature profile. The result of. these analyses indicate about $\pm 47 \%, \pm 29 \%$, and $\pm 11 \%$ uncertainty in the thermal conductance values for Ga-In-Sn, indium, and silver interstitial materials, respectively. Because a large fraction of the errors in the measurements is common to all cases, a smaller temperature difference across the interface (due to better conductance) results in larger fractional error and thus more uncertainty in thermal conductance. Thus, for highly conductive interfaces, substantially more accurate measurement and control system need to be utilized in order to obtain more precise results. For the present application of cooling optical substrates, the uncertainty in In-Ga-Sn thermal conductance does not constitute a significant uncertainty, because for very conductive interfaces, the bulk of temperature rise in the substrate is due to conductive resistance in the solid rather than convective resistance at the boundaries.

The results shown in Fig. 5 represent the average of several test runs. There are considerable variations in the thermal conductance values obtained from different runs but under identical conditions. These cannot be explained by system errors and are due to actual physical changes introduced between runs. Possible sources are changes in cylinder orientations with respect to each other, microscopic changes in cylinder surfaces during successive applications of pressure and heat, or interstitial material embedding in irregularities in the surface. 


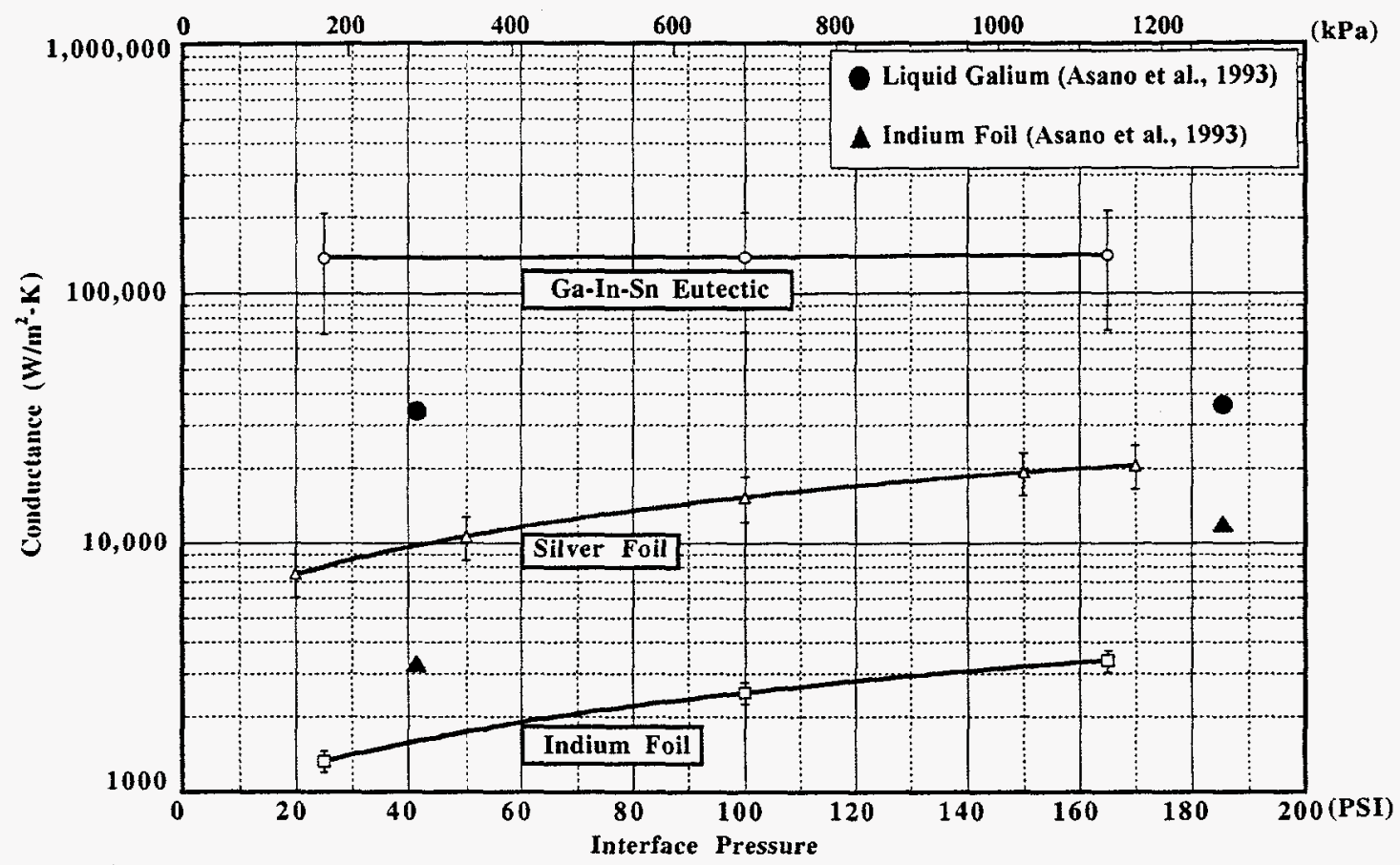

Figure 5: Variation of thermal contact conductance with pressure across a silicon-copper interface with indium foil, silver foil, or an In-Ga-Sn eutectic used as the interstitial material. Pressure is at $13 \mathrm{~Pa}$ ( 0.1 Torr) and average roughness of the copper and silicon surfaces are $0.07 \mu \mathrm{m}$ and $0.09 \mu \mathrm{m}$, respectively. Data presented are averages of several runs. For comparison, data from Asano et al. (1993) are also shown.

For comparison, included in Fig. 5 are the only data relevant to the present application that were found in the literature (Asano et al., 1993). The data were obtained in a similar experimental setup across an (apparently) silicon carbidecopper interface with gallium and indium used as the interstitial materials. An average roughness of $0.9 \mu \mathrm{m}$ for the contacting surfaces is quoted. No information on the experimental procedure, system calibration, repeatability of the results, flatness of the surfaces, etc., is given. It is thus difficult to make a comparison. These data as well as ours should be considered quantitative results, giving baseline information for contact-cooled mirror designs. If needed, more accurate results can be obtained post design, by measuring the time constant of a cooled mirror and estimating the contact conductance from that information. Even in this case, an area-averaged thermal contact conductance is obtained that ignores local variations caused by nonuniform applied pressure or surface figure and finish across the cooled area(s).

\section{ACKNOWLEDGMENTS}

The authors wish to acknowledge J. Arko, J. Attig, J. Grimmer, M. Sutton, and E. Trakhtenberg for their assistance. Fruitful discussions with M. M. Yovanovich from the University of Waterloo are acknowledged. This work supported by U.S. Department of Energy, BES-Material Sciences, under contract no. W-31-10-Eng-38.

\section{REFERENCES}

Asano, M, J. Ogata, and Y. Yosinaga, "Quantitative evaluation of contact thermal conductance in a vacuum as a result of simulating the effect of cooling," in High Heat Flux Engineering, Proc. SPIE 1793, pp. 652-656, 1993.

Kennard, E. H. Kinetic Theory of Gases, McGraw-Hill, New York, pp. 311-318, 1938.

Lambert, M. A., and Fletcher, L. S., "Review of Models for Thermal Contact Conductance of Metals," AIAA Journal of Thermophysics and Heat Transfer, Vol. 11, No. 2, pp. 129-140, 1997.

Yovanovich, M. M., and V. W. Antonetti, "Application of thermal contact resistance theory to electronic packages," in Advances in Thermal Modeling of Electronic Components and Systems, Vol. 1, A. Bar-Cohen and A. D. Kraus, Editors, pp. 79-128, Hemisphere Publishing Corp., New York, 1988. 